\section{Membrane fluidity matters: Hyperthermia from the aspects of lipids and membranes}

\author{
Balint Csoboz ${ }^{1}$, Gabor E. Balogh ${ }^{1}$, Erzsebet Kusz ${ }^{1}$, Imre Gombos ${ }^{1}$, Maria Peter ${ }^{1}$, Tim Crul $^{1}$, Burcin Gungor ${ }^{1}$, \\ Lajos Haracska ${ }^{2}$, Gordana Bogdanovics ${ }^{3}$, Zsolt Torok ${ }^{1}$, Ibolya Horvath ${ }^{1}$, \& Laszlo Vigh ${ }^{1}$ \\ ${ }^{1}$ Institute of Biochemistry, Biological Research Centre, Hungarian Academy of Sciences, Szeged, Hungary, ${ }^{2}$ Institute of Genetics, Biological Research \\ Centre, Hungarian Academy of Sciences, Szeged, Hungary, and ${ }^{3}$ Experimental Oncology Department, Oncology Institute of Vojvodina, \\ Put Dr Goldmana 4, 21204 Sremska Kamenica, Serbia
}

\section{Abstract}

Hyperthermia is a promising treatment modality for cancer in combination both with radio- and chemotherapy. In spite of its great therapeutic potential, the underlying molecular mechanisms still remain to be clarified. Due to lipid imbalances and 'membrane defects' most of the tumour cells possess elevated membrane fluidity. However, further increasing membrane fluidity to sensitise to chemo- or radiotherapy could have some other effects. In fact, hyperfluidisation of cell membrane induced by membrane fluidiser initiates a stress response as the heat shock protein response, which may modulate positively or negatively apoptotic cell death. Overviewing some recent findings based on a technology allowing direct imaging of lipid rafts in live cells and lipidomics, novel aspects of the intimate relationship between the 'membrane stress' of tumour cells and the cellular heat shock response will be highlighted. Our findings lend support to both the importance of membrane remodelling and the release of lipid signals initiating stress protein response, which can operate in tandem to control the extent of the ultimate cellular thermosensitivity. Overall, we suggest that the fluidity variable of membranes should be used as an independent factor for predicting the efficacy of combinational cancer therapies.

\section{Keywords}

Cancer therapy, heat shock protein, hyperthermia, lipid raft,membrane fluidity, thermotolerance

\section{History}

Received 27 March 2013

Revised 20 May 2013

Accepted 21 May 2013

Published online 1

\section{Introduction}

Hyperthermia, mainly as an adjuvant to radiotherapy and chemotherapy, is an established methodology among the currently applied cancer treatments. Although its exact mechanism is still unknown, it is one of the most effective radiation sensitisers and can additionally enhance the cytotoxicity of certain anticancer drugs [1]. Importantly, there is a tumour-selective effect of hyperthermia in a critical range of temperature $\left(40-43{ }^{\circ} \mathrm{C}\right)$ in vivo [2]. Various strategies and mechanisms underlying the clinical application of hyperthermia in combination with cancer immunotherapy have been discussed by Repasky et al. [3-5].

As a challenge to its therapeutic potential the use of hyperthermia in cancer therapy has an undesirable and inevitable side-effect linked to the familiar phenomenon in thermobiology known as acquisition of thermotolerance (ATT). A point relevant to the present review is that the subpopulation of cancer cells which develop thermotolerance become less sensitive to subsequent hyperthermia-induced cytotoxicity or various anticancer drugs and radiation [6]. Accordingly, the possibility of preventing thermotolerance

Correspondence: Laszlo Vigh, Institute of Biochemistry, Biological

59 Research Centre, Hungarian Academy of Sciences, H-6726 Szeged,

60 Hungary. Tel: 36-62-599-600/583. E-mail: vigh.laszlo@brc.mta.hu development by relatively harmless substances is of high 95 clinical importance.

Thermotolerance is generally associated with the synthesis and accumulation of heat shock proteins (HSP) molecular chaperones (especially Hsp70 and Hsp25/27). The exact sequence and mechanism of hyperthermia-induced events leading either to cell death or to the activation of cellular thermotolerance are still largely unexplored. [6]. Acquisition of thermotolerance is known to induce several other cellular defences, including the elevation of non-enzymatic and enzymatic antioxidants or activation the autophagy by NFKB during the phase of heat shock recovery [7].

As highlighted in this review, a large amount of evidence has also been presented for decades for the involvement of membranes both in the acquisition of thermotolerance and in heat lethality. As early as 1924, Heilbrunn proposed that the physical state of the lipids might be related to the extent of cell killing by heat [8]. Experimental evidences provided later by Yatvin and co-workers supported the hypothesis that the fluidity of membranes might be a major factor contributing to the death of mammalian cells exposed to hyperthermia [9].

Here first we briefly review those evidences, which show that the dysregulated lipid metabolism and membrane defects are really common in tumour cells, and that certain cancer therapies can alter the physicochemical properties of membranes. 
Then, based on a novel method which allowed the direct imaging of nanoscopic long-lived platforms with raft-like properties diffusing in the live cell plasma membrane, we discuss what the properties are that allow the surface membrane to become the key determinant of cellular heat stress sensing and signalling.

The next paragraph overviews how lipidomic fingerprints revealed that membrane stress achieved either by heat or membrane fluidiser benzyl alcohol (BA) results in highly specific alterations in lipid metabolism of melanoma cells. We emphasise how the activation of certain phospholipases coupled to the production of specific lipid mediators (such as arachidonic acid) can refine the expression of HSPs $[10,11]$.

Based on new studies next we provide evidence that isothermal membrane hyperfluidisation can induce an equal level of cellular thermotolerance with that achieved by heat priming, and both treatments are accompanied by specific remodelling of the microdomains of the surface membranes. The significantly lower levels of major HSPs (Hsp70, Hsp25) measured in membrane fluidiser treated cells also provided compelling evidence that the amount of HSPs produced is not the sole factor in the development of thermotolerance.

\section{Dysregulated lipid metabolism and membrane defects are common in tumour cells}

Alterations in lipid metabolism have long been recognised as a hallmark of cancer cells and are discussed in detail elsewhere [12]. As a consequence of this recognition a number of cancer drugs are currently under development or in the clinical phase targeting specific lipid metabolic pathways [13]. As suggested by Nomura et al., tumour cells undergo a general metabolic shift towards specific bioenergetic (glycolysis) and anabolic (protein and lipid synthesis) processes that promote rapid growth, and it was clearly demonstrated that an increase in the level of monoacylglycerol (MAG) lipase can drive tumorigenesis through the lipolytic release and remodelling of free fatty acids [14]. Since the influence of oncogene expression on the early lipidome alterations was unknown, we recently analysed the changes in the course of ERBB2 expression-mediated premature senescence induced in lipid profiles by using MCF-7 breast cancer cells. The most marked changes were found in the levels of PG (34:1), PG (36:1) (increased) and lysophosphatidylethanolamine (LPE) (18:1), phosphatidylglycerol (PG) (40:7) and phosphatidylinositol (PI) (36:1) (decreased). Statistical analysis revealed a general trend towards shortened phospholipid acyl chains in senescence, and these changes were accompanied by increased global membrane fluidity [15].

Several solid tumours are characterised by the higher fluidity of their cell membranes [16-20], correlating with their proliferative and invasive potentials and their metastatic abilities [21-23]. A reversal of tumour resistance to apoptotic stimuli through the alteration of membrane fluidity was suggested by Baritaki et al. [12]. Melanoma tumour cells with a high metastatic potential are characterised by an enhanced lateral mobility of the membrane receptors in metastasis, while exhibiting a reduced cholesterol/phospholipid ratio [24]. The plasma membrane (PM)-selective catalytic hydrogenation of lipids in live murine leukemic GRLS cells (i.e. an attempt to normalise bulk membrane fluidity by chemical means) notably increased the expression of a $15 \mathrm{kDa}$ antigen on the cell surface [25]. It was recently suggested that the ability of breast tumour stroma to promote the epithelialmesenchymal transition, the reduction of cell adhesion, the migration velocity and directness, and especially an increase in membrane fluidity, can be viewed as overall progressionand invasion-promoting effects [26].

\section{Cancer therapies can also alter the physicochemical properties of membranes: the case of cisplatin}

Tumour cells treated with cisplatin also exhibit an increase in PM fluidity, which results from the activation of acid sphingomyelinase and the subsequent generation of ceramide (Cer) [27]. The generation of Cer and the redistribution of the death receptor CD95 into the lipid rafts can promote the initiation of the apoptotic signal and the elimination of the malignant cells [27]. As will be discussed later, we have documented the accumulation of Cer both in heat- and in BA-pretreated B16 melanoma cells [10]. Most recent studies by Alvarez-Berrios et al. revealed that magnetic fluid hyperthermia combined with cisplatin resulted in significantly enhanced cytotoxicity when compared with hyperthermia using a water bath. It was shown that hyperthermic potentiation of cisplatin by magnetic nanoparticle heaters is correlated with an increase in the membrane fluidity, and as a consequence, elevated passive uptake of the drug in cancer cells. As was emphasised by the authors, the demonstrated mechanism in the context of cisplatin could find application in potentiation of other chemotherapies.

These and other findings urge a complete revision of our current concepts of the mode of action of platinum-based chemotherapy. The examination of transformation incidences expressed as a function of the surviving fraction revealed that the combination of heat and cisplatin resulted in fewer transformants per surviving cell than for cisplatin alone [28]. In other words, when heat converts sub-lethal damage to lethal damage in combination with cisplatin, the elevation of the membrane fluidity and the generation of Cer per se [10] can act synergistically as a 'common denominator' in hyperthermia and chemotherapy.

\section{Membranes are key determinants of cellular stress adaptation and lethality}

It was shown decades ago, that fluidity, organisation and phase behaviour of membranes are key and strictly controlled factors in the processes of thermally induced adaptation and lethality, in both prokaryotic [29-31] and eukaryotic cells [32-36]. Our early findings, achieved with prokaryotic models firstly revealed that membranes can act as thermosensors, and there exists a feed-back membrane fluidity control of certain stress defending genes, such as fatty acid desaturases in the cold [37]. But how do eukaryotic cells maintain the physical structure of their membrane lipid bilayers within optimal and/or tolerable limits? How changes in plasma membrane physical properties are perceived in a mammalian cell, and how the abundance of lipids in the plasma membrane is regulated to balance changing remains largely unknown. 
The plasma membrane (PM) in mammalian cells has been

hypothesised to contain nanoscopic lipid platforms, which are discussed in the context of 'lipid rafts' or 'membrane rafts'. The findings of biochemical and cell biological studies have prompted the belief that rafts play a crucial role in many signalling processes [38-40]. Since it has proven difficult to visualise rafts in living cells, there is currently no consensus on their size, shape, stability, surface density, composition and heterogeneity. Very recently, however, we introduced a method which allowed the direct imaging of nanoscopic longlived platforms with raft-like properties diffusing in the live cell PM [41]. This novel technique, called 'thinning out clusters while conserving the stoichiometry of labelling' (TOCCSL) can sense these platforms through their ability to assemble a characteristic set of fluorescent marker proteins or lipids on a time scale of milliseconds. A special photobleaching protocol was used to reduce the surface density of labelled mobile platforms down to the level of well-isolated diffraction-limited spots, without altering the single spot's brightness. The statistical distribution of probe molecules per platform was determined by single molecule brightness analysis. For demonstration we used the consensus raft marker glycosylphosphatidylinositol-anchored monomeric GFP (mGFP-GPI) and the fluorescent lipid analogue Bodipy-GM1, which preferentially partitions into liquid ordered phases. For both markers, we found a cholesteroldependent homo-association in the PM of living $\mathrm{CHO}$ and Jurkat $\mathrm{T}$ cells in the resting state, thereby demonstrating the existence of small, mobile, long-lived platforms containing these probes. We further applied this technology to address the structural changes in the PM during fever-type heat shock. At elevated temperatures, the mGFP-GPI homo-association disappeared, parallel with the increase in the expression of Hsp27. This finding lent strong support to our earlier suggestions that PM is involved in the sensing of temperature elevations through changes in the physical state of the membrane $[36,42,43]$. Interestingly, in artificial bilayer systems, atomic force microscopy studies have shown that GPI-anchored proteins can be released from the liquid ordered phase by an increase in temperature [44]. Thus, a similar mechanism may apparently account for the observed dissociation of mGFP-GPI homo-associates in the CHO cell membrane. Taken together, these findings provide direct support for our hypothesis that fever stress has the potential to remodel lipid rafts and, via modulating the membrane microdomains engaged in primary stress sensing and signalling, to enhance the expression of a distinct subclass of HSPs selectively.

By using the TOCCSL technology we next addressed how the combination of heat shock and a prominent HSP co-inducer drug candidate, hydroximic acid BGP-15 [45], affects the thermosensory properties of membranes. By using molecular dynamics simulations we provided evidence of the docking of BGP-15 in model membranes made of sphingomyelin-cholesterol. The specific interaction of BGP-15 with cholesterol (Chol) was further assessed by using a combination of complementary biophysical approaches. A reduced rate of Chol depletion by metyl-beta-cyclodextrin (MBCD) in the presence of BGP-15 was shown in vitro by the LangmuirBlodget monolayer technique. The above-described TOCCSL method allowed the direct imaging of raft integrity during 301 mild heat stress alone or in combination with the HSP co- 302 inducer BGP-15. Confocal microscopy allowed us to follow 303 the redistribution of Chol-rich membrane domains in response 304 to drug administration with the fPEG-Chol probe. It emerged 305 that BGP-15 partitions to lipid rafts with a preferential 306 affinity for Chol. Moreover, BGP-15 was able to remodel 307 Chol-enriched lipid platforms reminiscent of those observed 308 earlier following non-lethal heat priming or membrane stress, 309 and was shown to be obligatory for the generation and 310 transmission of stress signals [43]. The BGP-15 activation 311 of HSP expression involved the Rac1 signalling cascade. 312 Presumably via Rac1 (and other, as yet unrevealed signalling 313 pathways, bridging the signalling platforms of surface mem- 314 branes with $h s p$ genes via heat shock factors, (HSFs)), we 315 demonstrated that BGP-15 is able to inhibit the rapid HSF1 316 acetylation monitored during the early phase of heat stress, 317 thereby promoting a prolonged duration of HSF1 binding to 318 heat shock elements [46,47]. Modulation of the heat shock 319 protein response via drugs, such as BGP-15 acting on the base 320 of membrane lipid therapy has the potential to be beneficial 321 in a range of disorders, including cancer [36]. 322

\section{Lipidomics revealed membrane lipid remodelling and release of potential HSP-inducing lipid mediators during early stress responses in murine B16 melanoma cells}

Apart from their roles in the structural organisation of 329 membranes, different membrane lipids can be metabolised 330 and give rise to signalling molecules in response to various 331 stress stimuli. Increasing evidence (relating to sphingolipids 332 or phospholipase $A_{2}$ activation, for instance) links such 333 signalling processes to membrane microdomains. In turn the 334 lipid signalling molecules can alter the gene expression and 335 thereby couple environmental stress or other stimuli to energy 336 metabolism, cellular aging, for example. 337

With the aim of recognising lipid changes as a conse- 338 quence of heat shock and/or membrane fluidity modulation 339 achieved through administration of the non-proteotoxic BA, 340 we analysed the ESI-MS/MS molecular species data using 341 a data-mining principal component analysis method [10]. 342 This allowed a clear differentiation of the experiments into 343 four non-overlapping clusters, depending on the different 344 treatments. The first component, which accounted for almost 345 $60 \%$ of the variance, clearly distinguished the control and 346 stress conditions, suggesting that common lipid metabolic 347 pathways are involved in the stress-mediated lipid alterations. 348 The second and third components revealed differences 349 concerning mild or severe heat and the BA-induced mem- 350 brane stresses, indicating specific changes in the lipidome in 351 response to these membrane perturbations.

A key feature of the acute lipid remodelling due to heat 353 (both mild and severe) or BA-induced membrane perturbation 354 observed $60 \mathrm{~min}$ after stress intervention was the accumula- 355 tion of Chol, Cer and saturated PC and PE-P species in the 356 highly metastatic B16-F10 cells. These lipid species tend 357 to support the formation of tightly packed subdomains 358 corresponding to liquid-ordered phases biophysically char- 359 acterised in model membranes and raft domains in cells [48]. 360 
The altered microdomain disposition was confirmed by preliminary results of analysis of the lipid composition of detergent-resistant membrane domains (DRMs) from B16-F10 cells as a consequence of stress (Horvath et al., unpublished data). This indicated the recruitment of specific lipids into DRMs during membrane stress. It is known that elevated Cer levels can displace Chol from membrane/lipid'Chol-rafts' and form large, Cer-enriched membrane platforms 'Cer-rafts' [49,50]. Since both Chol and Cer (besides other raft-component lipids) accumulated during stress in whole B16-F10 cells, it is conceivable that the rafts undergo rearrangement and contain different protein components, thereby altering various signalling pathways, (such as those involving phosphatidylinositol 3-kinase, Akt and glycogen synthase kinase 3), which in turn may transmit the stress signal from the plasma membrane to the nucleus [35,51]. Taken together, these findings may explain our previous observations concerning heat- or BA-induced Chol-rich PM microdomain condensation observed by fluorescence microscopy in B16-F10 cells [43].

The increase in saturated lipids and the concomitant reduction of polyenes is a clear consequence of stress. This may highlight common metabolic processes which are involved in stress responses, whereas the lipid class- or the lipid species-dependent changes may reflect stressor-specific alterations. In accordance with the commonly accepted view [52-54] we suggest that the decrease in polyunsaturated fatty acid (PUFA)-containing lipids (with special emphasis on the 20:4-containing species) following heat and BA treatments is due to the action of phospholipases. Such enzymatic activity is thought to be influenced by membrane fluidity and/or microheterogeneity for both phospholipase $\mathrm{A}_{2}\left(\mathrm{PLA}_{2}\right)$ [55] and phospholipase C (PLC) [56]. These phospholipases are also known to be stimulated by heat shock (HS) and chemical stressors [57,58]. The lipid most affected by PUFA removal was PI (38:4), which can be metabolised mainly by PIspecific $\mathrm{PLA}_{2}$ [59] or by PLC. The latter also hydrolyses $\mathrm{PIP}_{2}$, thereby producing two second messengers, diacylglycerol (DAG) and inositol triphosphate $\left(\mathrm{IP}_{3}\right)[60] . \mathrm{IP}_{3}$ rapidly mediates the release of $\mathrm{Ca}^{2+}$ from the endoplasmic reticulum following binding to $\mathrm{IP}_{3}$ receptors. Interestingly, it has been reported that, in the initial stage of hyperthermia, the heat induces the turnover of polyphosphoinositides and the production of $\mathrm{Ca}^{2+}$-mobilising $\mathrm{IP}_{3}$ [61]. Moreover, a number of reports have indicated that HS leads to a rapid increase in the level of intracellular free $\mathrm{Ca}^{2+}$ from internal stores and a massive $\mathrm{Ca}^{2+}$ influx from the extracellular medium. Cell calcium appears to be critical for the transcriptional activation of $h s p$ genes in B16-F10 [43] and other cell lines [62].

Elevated activity of phosphoinositide-specific PLC results in the formation of DAG which is highly enriched in arachidonic acid (AA) and may therefore function as second messenger [63]. It could for example enhance the membrane association and activation of various isoforms of protein kinase $\mathrm{C}$ (PKC) which have been found to drive the phosphorylation of HSFs [51]. This is consistent with the induced expression of Hsp70s in response to the activation of PKC [35]. Moreover, the heat-induced accumulation of PS and the BA-induced enhancement of DAG may play a positive regulatory role in PKC activation and consequently in $\mathrm{HS}$ induction, since both PS and DAG are essential cofactors of 421 PKC $[64,65]$, but can be differently affected by the different 422 stressors. 20:4-DAG can be subsequently metabolised by 423 DAG lipase to 20:4- monoacylglycerol (20:4-MAG) [66,67] 424 after which AA can be released through the action of 425 monoacylglycerol lipase or fatty acid amide hydrolase action 426 [68]. AA released by both PLA 2 and PLC-mediated pathways 427 can mediate signal transduction and be recycled via the Lands 428 pathway, whereas a portion can be lost to $\beta$-oxidation. In fact, 429 the addition of AA to HeLa cells stimulated HSF1-DNA 430 binding, increased the phosphorylation of HSF1 and, up- 431 regulated the transcription of the $h s p 70$ gene [69] demonstrat- 432 ing its HSP modulator ability.

In line with the above findings, it was reasonable to assume 434 that the PUFA status and the ability of cells to respond to 435 stress are closely interconnected. The modulation in HSP 436 expression caused by plating density variation was studied by 437 Noonan et al. [70] who observed that the activation of two 438 human Hsp70 family members was indeed cell number- 439 dependent after heat shock in colon carcinoma cell lines. 440 As suggested by Koklic et al. in their 'membrane switch 441 hypothesis' [71], the cell density strongly influences the 442 lateral domain structure of tumour cell membranes by causing 443 the appearance or disappearance of certain membrane domain 444 types on the cell surface membranes (thereby acting as a 445 'switch'). We recently provided evidence that simply the 446 modulation of cell density considerably altered the induci- 447 bility of $h s p$ genes in B16-F10 cells, and was paralleled by 448 pronounced changes in both the Chol level and the size 449 distribution of pre-existing Chol-rich plasma membrane 450 rafts [46]. When B16-F10 melanoma cells were cultured at 451 different initial cell densities, lipidomic analysis revealed a 452 profound rearrangement of molecular species composition, 453 with around $70 \%$ of the lipid molecular species being altered. 454 At the same time, different culturing conditions dramatically 455 altered the stress inducibility of the major $h s p$ genes, $h s p 70 \quad 456$ and $h s p 25$ [11]. In general, the importance of our findings 457 lies in the need for n-3 and n-6 PUFA for the maintenance of 458 stress protein responsibility in mammalian cells, which cannot 459 synthesise their own, and draw attention to the need for their 460 careful control. In fact, tumour cells exhibit a pronounced 461 increase in de novo fatty acid synthesis, whereas normal cells 462 are thought to acquire fatty acids primarily from dietary 463 sources [72]. Moreover, our findings lend further support to 464 the importance of both the 'quality' of the pre-existing 465 membrane microdomains themselves and the release of lipid 466 mediators (such as AA and derivatives), together with other 467 stress protein-inducing signal transducers, which may act in 468 tandem to control the extent of the ultimate cellular stress 469 response [11]. $\quad 470$

A lipidomic approach will be useful for the determination 471 of lipidome changes with prospective value as biomarkers and 472 to disclose pathways with the potential for therapy [73,74]. 473 Furthermore, in order to understand the contribution of 474 membrane lipid composition to the functionality of mem- 475 brane-bound cellular processes (such as operation of surface 476 membrane receptors, ion channels, or the mitochondrial 477 electron transport chain), comprehensive structural and 478 quantitative information on the organellar lipidome is essen- 479 tial [75]. 
(A) 120

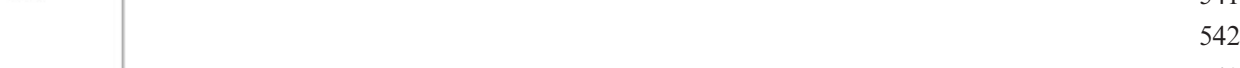

ஓి $100-1543$

ì 100

m 80

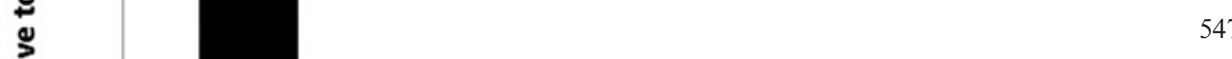

$\mathbf{0}$

(B)

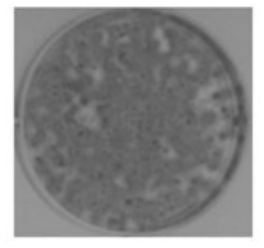

\section{Acquisition of thermotolerance via prior membrane} hyperfluidisation in B16-F10 melanoma cells

In a study by Balogh et al., the effects of the administration of the non-proteotoxic [42] membrane fluidiser BA and 'traditional' heat priming were investigated and compared concerning their capacities in thermotolerance development. B16-F10 cells were preconditioned $\left(40 \mathrm{mM} \mathrm{BA}\right.$ at $37^{\circ} \mathrm{C}$ or $42^{\circ} \mathrm{C}$ ) for $1 \mathrm{~h}$, and after a $16 \mathrm{~h}$ recovery period, were subjected to a $1 \mathrm{~h}$ lethal heat stress at $45^{\circ} \mathrm{C}$. As shown in Figure 1 , when preconditioned either with mild heat shock or with BA, these melanoma cells acquired a highly elevated thermotolerance. On the other hand, we observed a statistically insignificant difference in the effects induced by prior heat and isothermal membrane hyperfluidisation. Subsequently, we determined the level of the major HSPs, Hsp70 and Hsp25 by western blotting (Figure 2). Remarkably, whereas heat and BA priming exerted similar protective effects, BA treatment evoked weaker HSP response relative to heat priming at a HSP level. Noteworthy, no major differences were found in the levels of major HSPs between the heat sensitive B16 parent line and the heat resistant variants in other work, suggesting that HSPs are not a determining factor in the heatresistant phenotype of B16 melanoma cells [76]. Thus, the acquired heat tolerance observed in this study should involve other mechanisms (see above) rather than solely the de novo synthesis of HSP chaperones. Importantly, if applied at the concentration equipotent in membrane fluidisation with BA, pretreatment with phenethyl alcohol, shown to be ineffective as an $h s p$ activator, [43] also caused no measurable change of thermotolerance (unpublished observation).

It is noteworthy that we earlier demonstrated that the acquisition of cellular thermotolerance in BA-primed Escherichia coli cells was unrelated to the formation of the major HSPs, such as GroEL (Hsp60) and DnaK (Hsp70).
Instead, remodelling of the membrane lipid composition appeared to be sufficient for the development of short-term bacterial thermotolerance [30]. From studies using yeast unsaturated fatty acid auxotroph lipid mutants, Swan and Watson concluded that the strongly elevated heat sensitivity of unsaturated fatty acid-enriched cells is probably attributable to the membrane damage associated with increases in membrane fluidity independently of HSPs and trehaloze [77]. To unravel the possible mechanisms underlying the capability of BA for heat shock gene activation, we earlier revealed that, apart from membrane hyperfluidisation in the deep hydrophobic region, a distinct reorganisation of Chol-sphingomyelin-rich microdomains may also be required for the generation and transmission of stress signals to activate $h s p$ genes in B16 cells [43]. B16-F10 cells were next treated either with $40 \mathrm{mM}$ BA or heat-stressed at $42^{\circ} \mathrm{C}$ for $1 \mathrm{~h}$, and after a $16-\mathrm{h}$ recovery period incubated with the Bodipy $\mathrm{FL} \mathrm{C}_{5}$-sphingomyelin probe [78] for $10 \mathrm{~min}$. They were then washed and imaged with a custom-made ultrasensitive microscope in total internal reflection mode. The domain size was analysed with the freeware ImageJ software (www.uhnresearch.ca/ facilities/wcif/imagej), with its fast Fourier transform (FFT) bandpass filter and the nucleus counter plug-in (Figure 3). The sphingomyelin probe-labelled domains were sorted into six classes according to their diameters (Figure 3). Whereas the number of smaller domains decreased in response to both heat and BA priming, the larger domains accumulated. Importantly, the amplitude of the effects observed was always more pronounced in the case of BA-induced hyperfluidisation.

\section{HSPs are more than simply chaperones}

HSPs have multiple functions depending on their location. Some of the intracellular HSPs play an essential role as 
(A) 1,8

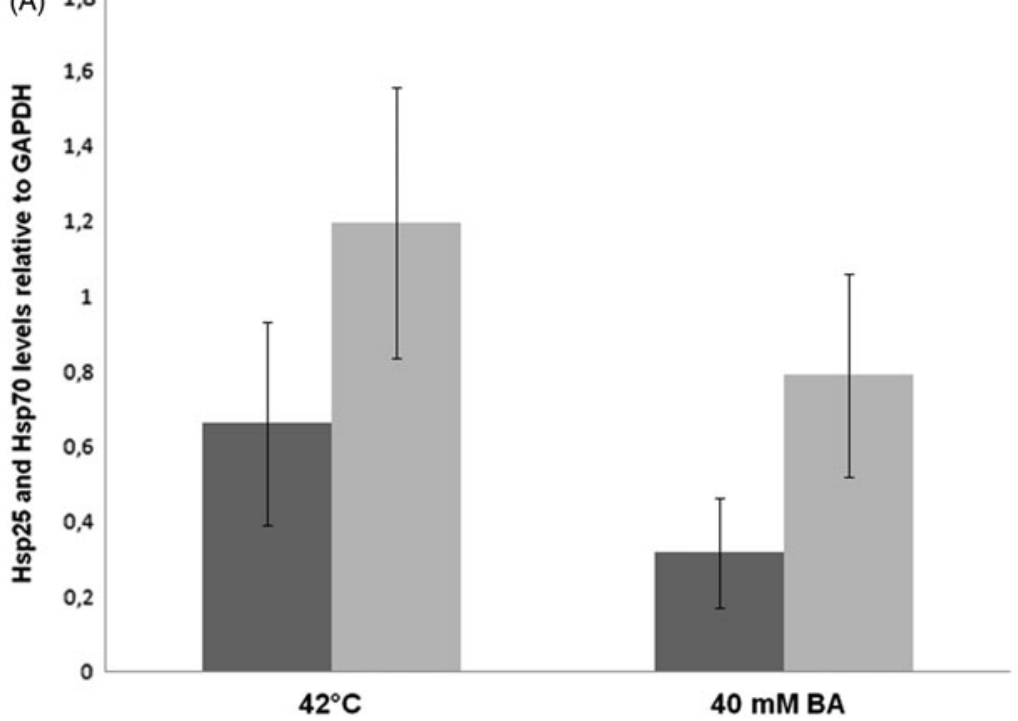

(B)

Hsp70

Hsp25

GAPDH
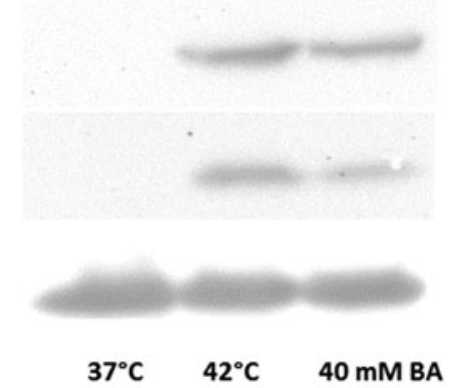

Figure 3. Redistribution of the cholesterolrich membrane domains on the surface of B16-F10 cells, monitored by a BODIPY FL C5 sphingomyelin probe.

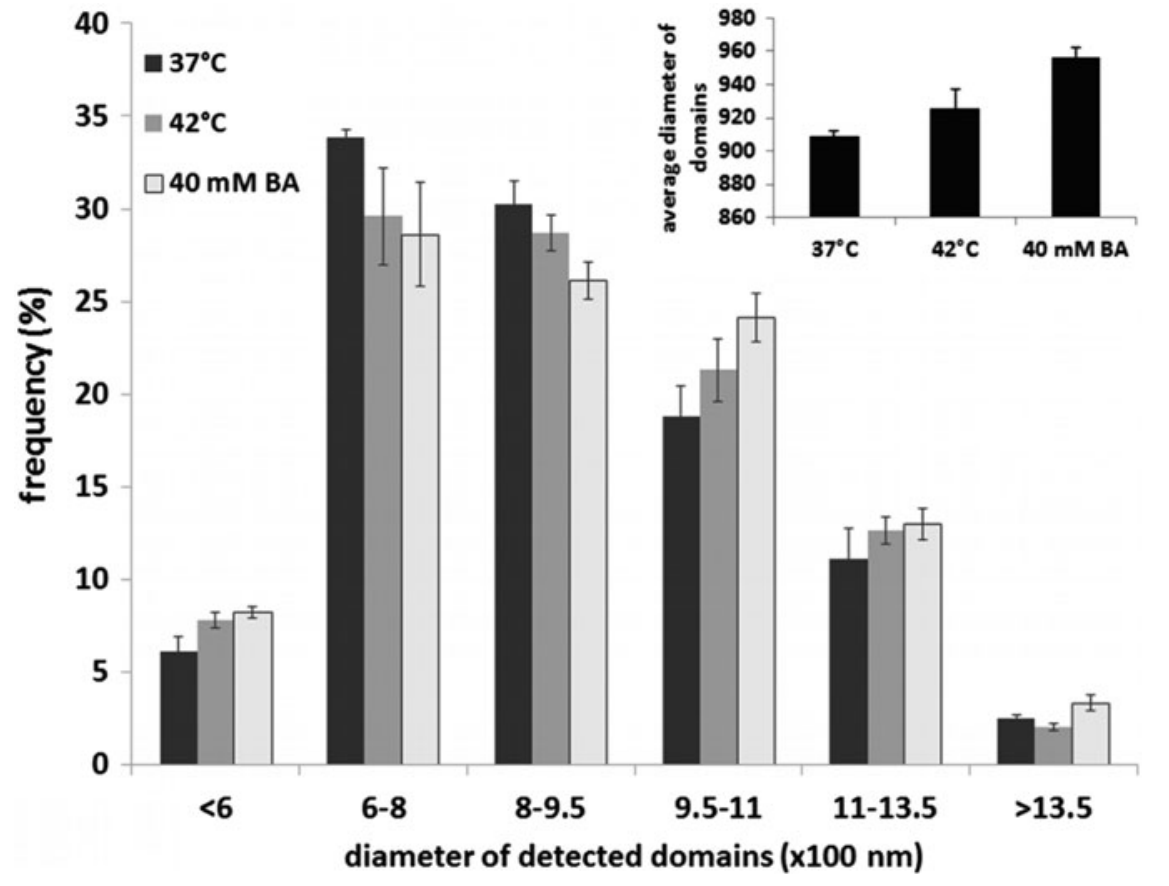

molecular chaperones by assisting the correct folding of nascent and stress-accumulated misfolded proteins, and preventing their aggregation. The protein- and/or lipidmediated association of a specific set of stress protein molecular chaperones to membranes is a widespread phenomenon that was earlier partially or completely overlooked, and is implicated in a number of physiological and pathological events $[64,79-81]$. Most relevant to the present review, temporary association of certain HSPs with membranes can reduce the level of fluidity [82-85], elevate bilayer stability [86], and thereby restore the membrane functionality during heat stress conditions. A novel $16.2 \mathrm{kDa}$ human small HSP, HspB11, was shown to inhibit $\mathrm{H}_{2} \mathrm{O}_{2}$, taxol and etoposideinduced cell death through preserving the integrity of the mitochondrial membrane system, the activation of Hsp90, the stabilisation of PM lipid rafts and activation of the PI-3kinase-Akt cytoprotective pathway. We recently provided evidence for the cholesterol-controlled interaction of HspB11 704 with lipid rafts [87].

Hsp70 interacts with an anionic phospholipid, 706 bis(monoacylglycero)phosphate, (BMP) that is predominantly 707 localised to the inner lysosomal membrane. The work of 708 Kirkegaard and co-workers confirmed [88] that the pH- 709 dependent (the interiors of lysosomes are highly acidic) and 710 high-affinity BMP-Hsp70 interaction strongly promotes cell 711 survival. The finding reveals a potential strategy for treating 712 cancer by inhibiting the lysosome-stabilising effects of Hsp70 713 in tumour cells, thereby promoting lysosome-dependent 714 autophagic cell death, in which the cell digests itself [81]. 715 So molecules that either inhibit Hsp70-related signalling 716 cascades (such as the PI3K/Akt/GSK pathway, which is 717 linked to up-regulated Hsp70 transcription in cancers [36]), 718 or drug candidates that directly block lysosomal localisation 719 of Hsp70, might prove useful in anticancer therapy [89]. 
The association with the plasma membrane seems to account for the pleiotropic effect of HSPs (predominantly small HSPs) which can contribute to the restoration of membrane activity following damage caused by abiotic stresses or cancer therapies. It is suggested that sHSPmediated membrane stabilisation precedes the thermal adaptation that occurs by adjustment of the lipid composition [83]. As we pointed out, the fluidity and microdomain organisation of membranes are decisive factors in the perception and transduction of stresses into signals that trigger the activation of specific heat shock genes [36]. Conversely, the membrane association of specific HSPs may result in the inactivation of membrane-perturbing signal(s), and thereby switch off the heat shock response. In that context, interactions between certain HSPs and specific lipid molecular species might be a previously unrecognised means for the compartmentalisation of HSPs to specific signalling platforms, where key stress signalling proteins are known to be concentrated.

Finally, the cancer metabolism can only be perceived as a network of pathways with plasticity, feedback loops and cross-talk that ensure the ultimate fitness of the tumour cells [90]. An understanding of the novel function of lipids and chaperones (free, membrane-bound or extracellular located) in the modulation of cell death and survival signalling, which is of fundamental importance in ATT, is just beginning to emerge. As suggested by Gabai and Sherman, the role of HSPs in the refolding of damaged proteins may not be as essential as earlier believed; instead, the role of HSPs is crucial in the regulation of signalling pathways [91]. Thus, the acquisition of further knowledge will be necessary in order to improve the therapeutic potential of hyperthermia.

\section{Concluding remarks}

Linked either to metabolic reprogramming or to therapy, the elevated extent of membrane fluidity and reorganisation of lipid rafts must be key determinants in the pleiotropic effects of hyperthermia leading ultimately to cellular adaptation or lethality. Comparative studies with heat- and membrane-primed melanoma cells reinforce the view that ATT involves general as well as stress-specific components. It is beyond doubt that, through their molecular chaperone activities, the prominent HSP family members can contribute to the development of thermotolerance. Further investigation of the role of membrane microdomain properties (biophysical and biochemical), together with the moonlighting HSPs in heat sensing, signalling and adaptation, and understanding the way these phenomena act as a network, appears essential to explore hyperthermia-induced events.

\section{Declaration of interest}

This study was supported by grants from the Hungarian Basic Research Fund (OTKA, no. 100857), by the Hungarian National Development Agency (TÁMOP-4.2.2/B-10/1-20100012) and by the Hungary-Serbia IPA Cross-Border Cooperation Programme (HUSRB/1002/214/126). The authors alone are responsible for the content and writing of the paper.

\section{References}

1. Pallepati P, Averill-Bates D. Mild thermotolerance induced at $40^{\circ} \mathrm{C}$ increases antioxidants and protects HeLa cells against mitochondrial apoptosis induced by hydrogen peroxide: Role of p53. Arch Biochem Biophys 2010;495:97-111.

2. Issels RD. Hyperthermia adds to chemotherapy. Eur J Cancer 2008; 44:2546-54.

3. Ostberg JR, Repasky EA. Emerging evidence indicates that physiologically relevant thermal stress regulates dendritic cell function. Cancer Immunol Immunother 2006;55:292-8.

4. Peer AJ, Grimm MJ, Zynda ER, Repasky EA. Diverse immune mechanisms may contribute to the survival benefit seen in cancer patients receiving hyperthermia. Immunol Res 2010;46:137-54.

5. Mace TA, Zhong L, Kilpatrick C, Zynda E, Lee C-T, Capitano M, et al. Differentiation of CD8 + T cells into effector cells is enhanced by physiological range hyperthermia. J Leukoc Biol 2011;90: 951-62.

6. Takahashi A, Yamakawa N, Mori E, Ohnishi K, Yokota S, Sugo N, et al. Development of thermotolerance requires interaction between polymerase-beta and heat shock proteins. Cancer Sci 2008;99: 973-8.

7. Nivon M, Richet E, Codogno P, Arrigo A-P, Kretz-Remy C. Autophagy activation by NFkappaB is essential for cell survival after heat shock. Autophagy 2009;5:766-83.

8. Heilbrunn, LV. The colloid chemistry of protoplasm. IV. The heat coagulation of protoplasm. Am J Physiol 1924;69:190-9.

9. Yatvin MB, Clifton KH, Dennis WH. Hyperthermia and local anesthetics: Potentiation of survival of tumor-bearing mice. Science 1979;205:195-6.

10. Balogh G, Péter M, Liebisch G, Horváth I, Török Z, Nagy E, et al. Lipidomics reveals membrane lipid remodelling and release of potential lipid mediators during early stress responses in a murine melanoma cell line. Biochim Biophys Acta 2010;1801: 1036-47.

11. Péter M, Balogh G, Gombos I, Liebisch G, Horváth I, Török Z, et al. Nutritional lipid supply can control the heat shock response of B16 melanoma cells in culture. Mol Membr Biol 2012;29:274-89.

12. Baritaki S, Apostolakis S, Kanellou P, Dimanche-Boitrel M-T, Spandidos DA, Bonavida B. Reversal of tumor resistance to apoptotic stimuli by alteration of membrane fluidity: Therapeutic implications. Adv Cancer Res 2007;98:149-90.

13. Zhang F, Du G. Dysregulated lipid metabolism in cancer. World J Biol Chem 2012;3:167-74.

14. Nomura DK, Long JZ, Niessen S, Hoover HS, Ng S-W, Cravatt BF. Monoacylglycerol lipase regulates a fatty acid network that promotes cancer pathogenesis. Cell 2010;140:49-61.

15. Cadenas C, Vosbeck S, Hein E-M, Hellwig B, Langer A, Hayen H, et al. Glycerophospholipid profile in oncogene-induced senescence. Biochim Biophys Acta 2012;1821:1256-68.

16. Funaki N, Tanaka J, Kono Y, Nonaka A, Yotsumoto F, Lee J-U, et al. Combination of alpha-fetoprotein mRNA-based detection of hematogenously disseminating hepatocellular carcinoma cells and analysis of cancer cell membrane fluidity is more accurate in screening patients at risk of postoperative recurrence. Oncol Rep 2004;11:637-9.

17. Sok M, Sentjurc M, Schara M, Stare J, Rott T. Cell membrane fluidity and prognosis of lung cancer. Ann Thorac Surg 2002;73: 1567-71.

18. Yukihara M, Komizu Y, Tanoue O, Matsushita T, Matsumoto Y, Ueoka R. Specific accumulation and antitumor effects of hybrid liposomes on the growth of lung tumor cells. Yakugaku Zasshi 2010;130:1581-7.

19. Batko J, Płotast-Necas B, Warchoł T, Karon H. The effect of an experimental neoplastic disease on the flux of sodium and potassium ions across red blood cells and on the lipid composition of their membranes. Acta Biochim Pol 1992;39:317-26.

20. Barker CJ, Bowler K. Lipid composition of the membranes from cells of two rat tumors and its relationship to tumor thermosensitivity. Radiat Res 1991;125:48-55.

21. Gonda K, Watanabe TM, Ohuchi N, Higuchi H. In vivo nanoimaging of membrane dynamics in metastatic tumor cells using quantum dots. J Biol Chem 2010;285:2750-7.

22. Zeisig R, Koklic T, Wiesner B, Fichtner I, Sentjurc M. Increase in fluidity in the membrane of MT3 breast cancer cells correlates 
with enhanced cell adhesion in vitro and increased lung metastasis in NOD/SCID mice. Arch Biochem Biophys 2007;459:98-106.

23. Taraboletti G, Perin L, Bottazzi B, Mantovani A, Giavazzi R, Salmona M. Membrane fluidity affects tumor-cell motility, invasion and lung-colonizing potential. Int J Cancer 1989;44:707-13.

24. Sherbet G V. Membrane fluidity and cancer metastasis. Exp Cell Biol 1989;57:198-205.

25. Benkö S, Hilkmann H, Vigh L, Van Blitterswijk WJ. Catalytic hydrogenation of fatty acyl chains in plasma membranes: Effect on membrane lipid fluidity and expression of cell surface antigens. Biochim Biophys Acta 1987;896:129-35.

26. Angelucci C, Maulucci G, Lama G, Proietti G, Colabianchi A, Papi M, et al. Epithelial-stromal interactions in human breast cancer: Effects on adhesion, plasma membrane fluidity and migration speed and directness. PloS One 2012;7:e50804.

27. Segui B, Legembre P. Redistribution of CD95 into the lipid rafts to treat cancer cells? Recent Pat Anticancer Drug Discov 2010;5: $22-8$.

28. Miller RC, Richards M, Baird C, Martin S, Hall EJ. Interaction of hyperthermia and chemotherapy agents: Cell lethality and oncogenic potential. Int J Hyperthermia 1994;10:89-99.

29. Horváth I, Glatz A, Varvasovszki V, Török Z, Páli T, Balogh G, et al. Membrane physical state controls the signaling mechanism of the heat shock response in Synechocystis PCC 6803: Identification of $h s p 17$ as a 'fluidity gene'. Proc Natl Acad Sci USA 1998;95: 3513-18.

30. Shigapova N, Török Z, Balogh G, Goloubinoff P, Vígh L, Horváth I. Membrane fluidization triggers membrane remodeling which affects the thermotolerance in Escherichia coli. Biochem Biophys Res Commun 2005;328:1216-23.

31. Horváth I, Glatz A, Nakamoto H, Mishkind ML, Munnik T, Saidi $\mathrm{Y}$, et al. Heat shock response in photosynthetic organisms: Membrane and lipid connections. Prog Lipid Res 2012;51:208-20.

32. Vigh L, Horváth I, Horváth LI, Dudits D, Farkas T. Protoplast plasmalemma fluidity of hardened wheats correlates with frost resistance. FEBS Lett 1979;107:291-4.

33. Vigh L, Horváth I, Farkas T, Horvátht LI, Belea A. Adaptation of membrane fluidity of rye wheat seedlings according to temperature. Phytochemistry 1979;18:787-9.

34. Vigh L, Maresca B, Harwood JL. Does the membrane's physical state control the expression of heat shock and other genes? Trends Biochem Sci 1998;23:369-74.

35. Vigh L, Escribá P V, Sonnleitner A, Sonnleitner M, Piotto S, Maresca B, et al. The significance of lipid composition for membrane activity: New concepts and ways of assessing function. Prog Lipid Res 2005;44:303-44.

36. Vigh L, Horváth I, Maresca B, Harwood JL. Can the stress protein response be controlled by 'membrane-lipid therapy'? Trends Biochem Sci 2007;32:357-63.

37. Vigh L, Los DA, Horváth I, Murata N. The primary signal in the biological perception of temperature: Pd-catalyzed hydrogenation of membrane lipids stimulated the expression of the desA gene in Synechocystis PCC6803. Proc Natl Acad Sci USA 1993;90: 9090-4.

38. Simons K, Sampaio JL. Membrane organization and lipid rafts. Cold Spring Harb Perspect Biol 2011;3(10):a004697.

39. Olivera-Couto A, Aguilar PS. Eisosomes and plasma membrane organization. Mol Genet Genomics 2012;287:607-20.

40. Balogh G, Péter M, Glatz A, Gombos I, Török Z, Horváth I, et al. Key role of lipids in heat stress management. FEBS Lett 2013;587:1970-80.

41. Brameshuber M, Weghuber J, Ruprecht V, Gombos I, Horváth I, Vigh L, et al. Imaging of mobile long-lived nanoplatforms in the live cell plasma membrane. J Biol Chem 2010;285:41765-71.

42. Balogh G, Horváth I, Nagy E, Hoyk Z, Benkõ S, Bensaude O, et al. The hyperfluidization of mammalian cell membranes acts as a signal to initiate the heat shock protein response. FEBS J 2005;272: $6077-86$.

43. Nagy E, Balogi Z, Gombos I, Akerfelt M, Björkbom A, Balogh G, et al. Hyperfluidization-coupled membrane microdomain reorganization is linked to activation of the heat shock response in a murine melanoma cell line. Proc Natl Acad Sci USA 2007;104: 7945-50.

44. Giocondi M-C, Besson F, Dosset P, Milhiet P-E, Le Grimellec C. Temperature-dependent localization of GPI-anchored intestinal alkaline phosphatase in model rafts. J Mol Recognit 2007;20: 531-7.

45. Chung J, Nguyen A-K, Henstridge DC, Holmes AG, Chan MHS, Mesa JL, et al. HSP72 protects against obesity-induced insulin resistance. Proc Natl Acad Sci USA 2008;105:1739-44.

46. Gombos I, Crul T, Piotto S, Güngör B, Török Z, Balogh G, et al. Membrane-lipid therapy in operation: The HSP co-inducer BGP-15 activates stress signal transduction pathways by remodeling plasma membrane rafts. PLOS One 2011;6(12):e28818.

47. Crul T, Toth N, Piotto S, Literati-Nagy P, Tory K, Haldimann P, et al. Hydroximic acid derivatives: Pleiotropic HSP co-inducers restoring homeostasis and robustness. Curr Pharm Des 2013;19: 309-46.

48. Mukherjee S, Maxfield FR. Membrane domains. Annu Rev Cell Dev Biol 2004:20:839-66.

49. Grassme H, Riethmuller J, Gulbins E, Grassmé H, Riethmüller J. Biological aspects of ceramide-enriched membrane domains. Prog Lipid Res 2007;46:161-70.

50. Patra SK. Dissecting lipid raft facilitated cell signaling pathways in cancer. Biochim Biophys Acta 2008;1785:182-206.

51. Vigh L, Nakamoto H, Landry J, Gomez-Munoz A, Harwood JL, Horvath I. Membrane regulation of the stress response from prokaryotic models to mammalian cells. Ann N Y Acad Sci 2007; 1113:40-51

52. Balboa MA, Balsinde J. Oxidative stress and arachidonic acid mobilization. Biochim Biophys Acta 2006;1761:385-91.

53. Balsinde J, Winstead M V, Dennis EA. Phospholipase A(2) regulation of arachidonic acid mobilization. FEBS Lett 2002 531:2-6.

54. Hirabayashi T, Murayama T, Shimizu T. Regulatory mechanism and physiological role of cytosolic phospholipase A2. Biol Pharm Bull 2004;27:1168-73.

55. Hønger T, Jørgensen K, Biltonen RL, Mouritsen OG. Systematic relationship between phospholipase A2 activity and dynamic lipid bilayer microheterogeneity. Biochemistry 1996;35:9003-6.

56. Ahyayauch H, Villar A V, Alonso A, Goñi FM. Modulation of PI-specific phospholipase $\mathrm{C}$ by membrane curvature and molecula order. Biochemistry 2005;44:11592-600.

57. Calderwood SK, Stevenson MA, Price BD. Activation of phospholipase $\mathrm{C}$ by heat shock requires GTP analogs and is resistant to pertussis toxin. J Cell Physiol 1993;156:153-9.

58. Samples BL, Pool GL, Lumb RH. Polyunsaturated fatty acids enhance the heat induced stress response in rainbow trout (Oncorhynchus mykiss) leukocytes. Comp Biochem Physiol B Biochem Mol Biol 1999;123:389-97.

59. Corda D, Zizza P, Varone A, Filippi BM, Mariggiò S The glycerophosphoinositols: Cellular metabolism and biologica functions. Cell Mol Life Sci 2009;66:3449-67.

60. Wymann MP, Schneiter R. Lipid signalling in disease. Nat Rev Mol Cell Biol 2008;9:162-76.

61. Calderwood SK, Stevenson MA, Hahn GM. Heat stress stimulates inositol trisphosphate release and phosphorylation of phosphoinositides in CHO and Balb C 3T3 cells. J Cell Physiol 1987;130: 369-76.

62. Kiang JG, Tsokos GC. Heat shock protein $70 \mathrm{kDa}$ : Molecular biology, biochemistry, and physiology. Pharmacol Ther 1998;80: 183-201.

63. Wakelam MJ. Diacylglycerol - When is it an intracellular messenger? Biochim Biophys Acta 1998;1436:117-26.

64. Escribá P V, González-Ros JM, Goñi FM, Kinnunen PKJ, Vigh L, Sánchez-Magraner L, et al. Membranes: A meeting point for lipids, proteins and therapies. J Cell Mol Med 2008;12:829-75.

65. Griner EM, Kazanietz MG. Protein kinase C and other diacylgly cerol effectors in cancer. Nat Rev Cancer 2007;7:281-94.

66. Shimizu T, Yokotani K. Bidirectional roles of the brain 2-arachidonoyl-sn-glycerol in the centrally administered vasopressin-induced adrenomedullary outflow in rats. Eur J Pharmaco 2008:582:62-9.

67. Tang X, Edwards EM, Holmes BB, Falck JR, Campbell WB. Role of phospholipase $\mathrm{C}$ and diacylglyceride lipase pathway in arachidonic acid release and acetylcholine-induced vascular relaxation in rabbit aorta. Am J Physiol Heart Circ Physiol 2006;290: H37-45.

68. Chau LY, Tai HH. Release of arachidonate from diglyceride in human platelets requires the sequential action of a diglyceride 
lipase and a monoglyceride lipase. Biochem Biophys Res Commun 1981;100:1688-95.

69. Jurivich DA, Pachetti C, Qiu L, Welk JF. Salicylate triggers heat shock factor differently than heat. J Biol Chem 1995;270:24489-95.

70. Noonan EJ, Place RF, Rasoulpour RJ, Giardina C, Hightower LE. Cell number-dependent regulation of Hsp70B' expression: Evidence of an extracellular regulator. J Cell Physiol 2007;210: 201-11.

71. Koklic T, Pirs M, Zeisig R, Abramović Z, Sentjurc M. Membrane switch hypothesis. 1. Cell density influences lateral domain structure of tumor cell membranes. J Chem Inf Model 2005;45: 1701-7.

72. Menendez JA, Lupu R. Fatty acid synthase and the lipogenic phenotype in cancer pathogenesis. Nat Rev Cancer 2007;7:763-77.

73. Hønger T, Jørgensen K, Biltonen RL, Mouritsen OG. Systematic relationship between phospholipase A2 activity and dynamic lipid bilayer microheterogeneity. Biochemistry 1996;35:9003-6.

74. Fernandis AZ, Wenk MR. Lipid-based biomarkers for cancer. J Chromatogr B Analyt Technol Biomed Life Sci 2009;877:2830-5.

75. Klose C, Surma MA, Simons K. Organellar lipidomics-background and perspectives. Curr Opin Cell Biol 2013. doi: 10.1016/ j.ceb.2013.03.005.

76. Anderson RL, Tao TW, Betten DA, Hahn GM. Heat shock protein levels are not elevated in heat-resistant B16 melanoma cells. Radiat Res 1986;105:240-6.

77. Swan TM, Watson K. Stress tolerance in a yeast lipid mutant: membrane lipids influence tolerance to heat and ethanol independently of heat shock proteins and trehalose. Can J Microbiol 1999; 45:472-9.

78. Redman CA, Kennington S, Spathopoulou T, Kusel JR. Interconversion of sphingomyelin and ceramide in adult Schistosoma mansoni. Mol Biochem Parasitol 1997;90:145-53.

79. Nakamoto H, Vigh L. The small heat shock proteins and their clients. Cell Mol Life Sci 2007;64:294-306.

80. Horváth I, Multhoff G, Sonnleitner A, Vígh L. Membraneassociated stress proteins: More than simply chaperones. Biochim Biophys Acta 2008;1778:1653-64.

81. Horváth I, Vígh L. Cell biology: Stability in times of stress. Nature 2010;463:436-8.
82. Török Z, Horváth I, Goloubinoff P, Kovács E, Glatz A, Balogh G, et al. Evidence for a lipochaperonin: Association of active protein-folding GroESL oligomers with lipids can stabilize membranes under heat shock conditions. Proc Natl Acad Sci USA 1997; 94:2192-7.

83. Török Z, Goloubinoff P, Horváth I, Tsvetkova NM, Glatz A, Balogh $\mathrm{G}$, et al. Synechocystis Hsp17 is an amphitropic protein that stabilizes heat-stressed membranes and binds denatured proteins for subsequent chaperone-mediated refolding. Proc Natl Acad Sci USA 2001;98:3098-103.

84. Balogi Z, Török Z, Balogh G, Jósvay K, Shigapova N, Vierling E, et al. Heat shock lipid in cyanobacteria during heat/lightacclimation. Arch Biochem Biophys 2005;436:346-54.

85. Balogi Z, Cheregi O, Giese KC, Juhász K, Vierling E, Vass I, et al. A mutant small heat shock protein with increased thylakoid association provides an elevated resistance against UV-B damage in Synechocystis 6803. J Biol Chem 2008;283:22983-91.

86. Tsvetkova NM, Horváth I, Török Z, Wolkers WF, Balogi Z, Shigapova N, et al. Small heat-shock proteins regulate membrane lipid polymorphism. Proc Natl Acad Sci USA 2002;99:13504-9.

87. Török Z, Pilbat AM, Gombos I, Hocsák E, Sümegi B, Horváth I, et al. Evidence on cholesterol-controlled lipid raft interaction of the small heat shock protein HSPB11. In: Henderson B, Pockley AG, editors. Cellular Trafficking of Cell Stress Proteins in Health and Disease. Dordrecht: Springer; 2012.

88. Kirkegaard T, Roth AG, Petersen NHT, Mahalka AK, Olsen OD, Moilanen I, et al. Hsp70 stabilizes lysosomes and reverts NiemannPick disease-associated lysosomal pathology. Nature 2010;463: 549-53.

89. Juhász K, Thuenauer R, Spachinger A, Duda E, Horváth I, Vígh L, et al. Lysosomal rerouting of Hsp70 trafficking as a potential immune activating tool for targeting melanoma. Curr Pharm Des 2013;19:430-40.

90. Carracedo A, Cantley LC, Pandolfi PP. Cancer metabolism: Fatty acid oxidation in the limelight. Nat Rev Cancer 2013;13:227-32.

91. Gabai VL, Sherman MY. Invited review: Interplay between molecular chaperones and signaling pathways in survival of heat shock. J Appl Physiol 2002;92:1743-8. 